\title{
Spectrally decomposed dark-to-light transitions in Synechocystis sp. PCC 6803
}

\author{
Alonso M. Acuña ${ }^{1}$ (1) $\cdot$ Pascal van Alphen ${ }^{2} \cdot$ Filipe Branco dos Santos $^{2} \cdot$ Rienk van Grondelle $^{1} \cdot$ Klaas J. Hellingwerf $^{2}$. \\ Ivo H. M. van Stokkum ${ }^{1}$ (1)
}

Received: 1 November 2017 / Accepted: 23 March 2018 / Published online: 29 March 2018

(c) The Author(s) 2018

\begin{abstract}
Photosynthetic activity and respiration share the thylakoid membrane in cyanobacteria. We present a series of spectrally resolved fluorescence experiments where whole cells of the cyanobacterium Synechocystis sp. PCC 6803 and mutants thereof underwent a dark-to-light transition after different dark-adaptation (DA) periods. Two mutants were used: (i) a PSI-lacking mutant ( $\triangle \mathrm{PSI}$ ) and (ii) M55, a mutant without NAD(P)H dehydrogenase type-1 (NDH-1). For comparison, measurements of the wild-type were also carried out. We recorded spectrally resolved fluorescence traces over several minutes with $100 \mathrm{~ms}$ time resolution. The excitation light was at $590 \mathrm{~nm}$ so as to specifically excite the phycobilisomes. In $\triangle$ PSI, DA time has no influence, and in dichlorophenyl-dimethylurea (DCMU)-treated samples we identify three main fluorescent components: PB-PSII complexes with closed (saturated) RCs, a quenched or open PB-PSII complex, and a PB-PSII 'not fully closed.' For the PSI-containing organisms without DCMU, we conclude that mainly three species contribute to the signal: a PBPSII-PSI megacomplex with closed PSII RCs and (i) slow PB $\rightarrow$ PSI energy transfer, or (ii) fast PB $\rightarrow$ PSI energy transfer and (iii) complexes with open (photochemically quenched) PSII RCs. Furthermore, their time profiles reveal an adaptive response that we identify as a state transition. Our results suggest that deceleration of the PB $\rightarrow$ PSI energy transfer rate is the molecular mechanism underlying a state 2 to state 1 transition.
\end{abstract}

Keywords Cyanobacteria - Spectrally resolved fluorometry · Singular value decomposition · Time-resolved spectroscopy · Plastoquinone pool $\cdot$ Cyclic electron flow

$\begin{array}{ll}\text { Abbreviations } \\ \text { ATP } & \text { Adenosine triphosphate } \\ \text { CEF } & \text { Cyclic electron flow } \\ \text { COX } & \text { Cytochrome } c \text { oxidase } \\ \text { DA } & \text { Dark adaptation }\end{array}$

Alonso M. Acuña and Pascal van Alphen have contributed equally to this work.

\section{Electronic supplementary material The online version of this} article (https://doi.org/10.1007/s11120-018-0505-0) contains supplementary material, which is available to authorized users.

Ivo H. M. van Stokkum

i.h.m.van.stokkum@vu.nl

http://www.nat.vu.nl/ ivo/

1 LaserLaB, Department of Physics and Astronomy, Faculty of Science, Vrije Universiteit Amsterdam, De Boelelaan 1081, 1081 HV Amsterdam, The Netherlands

2 Swammerdam Institute for Life Sciences, University of Amsterdam, 1098 XH Amsterdam, The Netherlands

$\begin{array}{ll}\text { DCMU } & \text { Dichlorophenyl-dimethylurea } \\ \text { EET } & \text { Excitation energy transfer } \\ \text { FDP } & \text { Flavodiiron proteins } \\ \text { FNR } & \text { Ferredoxin-NADP + reductase } \\ \text { Flv1/3 (2/4) } & \text { Flavodiiron protein 1/3 (2/4) } \\ F_{\text {M }} & \text { Maximum fluorescence during a saturating } \\ & \text { pulse } \\ F_{\mathrm{S}} & \text { Steady-state fluorescence at the end of back- } \\ & \text { ground illumination } \\ F_{V} & \text { Variable fluorescence } \\ \text { LEF } & \text { Linear electron flow } \\ \text { HliP } & \text { High-light inducible protein } \\ \text { NADPH } & \text { Nicotinamide adenine dinucleotide } \\ & \text { phosphate } \\ \text { NDH-1 } & \text { NAD(P)H dehydrogenase type-1 } \\ \text { PB } & \text { Phycobilisome } \\ \text { PC } & \text { Plastocyanin } \\ \text { PMF } & \text { Proton-motive force } \\ \text { PQ } & \text { Plastoquinone } \\ \text { PS } & \text { Photosystem }\end{array}$


SAS

SDH Species-associated spectrum

SVD

WT
Singular value decomposition

Wild-type

\section{Introduction}

Cyanobacteria possess a remarkable thylakoid membrane that contains both photosynthetic and respiratory subunits. Photosynthetic activity is best described by the so-called $\mathrm{Z}$ scheme (Govindjee and Shevela 2011; Björn and Nickelsen 2013; Stirbet and Govindjee 2011). Input light interacts with pigment-protein complexes, the phycobilisomes (PB), that absorb and transfer excitation energy downhill to a series of cofactors until it reaches a particular pair of chlorophylls (Chls), the special pair, located in the core pigment-protein complexes of photosynthesis: the photosystems (PS) I and II. The special pair is excited to a charge transfer state (van Grondelle et al. 1994) from which an electron is released and, after being itself transferred through a series of cofactors, including primary electron acceptor $Q_{\mathrm{A}}$, it is expelled from PSII in the form of the twice reduced secondary electron acceptor $Q_{\mathrm{B}}\left(\mathrm{PQH}_{2}\right)$ that physically wanders to the cytochrome $b_{6} f$ (cyt $\left.b_{6} f\right)$. From there, electrons are shuttled to a small copper containing protein, the plastocyanin (PC) that can deliver the electron to PSI. This, so-called, linear electron flow (LEF) finalizes with the electron transfer from PSI to ferredoxin (Fd) and to the ferredoxin-NADP + reductase (FNR) which in turn catalyzes the reduction of $\mathrm{NADP}^{+}$ to NADPH (van Thor et al. 1999).

Remarkably, in cyanobacteria respiratory processes share some of the same components, as the plastoquinone (PQ) pool, the PC pool, the cyt $b_{6} f$ and the cytochrome oxidase participate both in photosynthetic and respiratory activities (Liu et al. 2012; Liu 2015; Lea-Smith et al. 2016; Schmetterer 1994; Jeanjean et al. 1993). The respiratory subunits providing the electron input to the PQ pool are the type-1 and -2 NADPH dehydrogenase, respectively, NDH-1 and $\mathrm{NDH}-2$, and the succinate dehydrogenase (SDH). NDH-1 and SDH have been reported to be the main sites where electrons are donated (Liu et al. 2012; Cooley and Vermaas 2001). As for LEF, $\mathrm{PQH}_{2}$ delivers the electrons to cyt $b_{6} f$ from where they either continue their way to the cytochrome oxidase via the PC pool and accomplish respiration or, alternatively, get shuttled to PSI and participate in cyclic electron flow (CEF) around PSI, a mechanism by means of which the cell adjusts the NADPH/ATP production ratio. Thus, as light input drops and photosynthetic electron flow stops, the PQ pool still serves as a vehicle for electron transfer related to ongoing respiratory activity.

Furthermore, it has been reported that the flavodiiron proteins (FDPs) provide alternative electron transfer pathways

(Shimakawa et al. 2015; Ermakova et al. 2016; Zhang et al. 2009; Allahverdiyeva et al. 2013) and are involved in photoprotection (Allahverdiyeva et al. 2015; Zhang et al. 2009; Bersanini et al. 2017) although the specific function of these heterodimers remains a controversial topic. The cyanobacterium Synechocystis sp. PCC 6803 (Synechocystis hereafter) has four FDPs: Flv1-4. While the pair Flv1/3 is involved in photoreduction of $\mathrm{O}_{2}$ (Allahverdiyeva et al. 2013) after accepting electrons from PSI, Zhang et al. (2009) reported plummeting levels of evolved oxygen in a mutant of Synechocystis lacking Flv2/4 and exposed to high-light, making them more susceptible to photoinhibition, and therefore, suggesting that Flv2/4 can supply PSII with an additional channel for electron transfer to alleviate excitation pressure. Crucially, Bersanini et al. (2014) found that overexpression of the flv4-2 operon in Synechocystis led to improved PSII photochemistry and kept the PQ pool in a predominantly oxidized redox state, providing unequivocal evidence that Flv2/4 acts as an important electron sink at the PSII acceptor side. The authors also report that a fully assembled phycobilisome is required for the stable expression of the electron sink mechanism.

Extended periods (several minutes) of darkness are known to drive the cyanobacterial cell towards a low fluorescence state denominated state 2 (Mullineaux and Allen 1986, 1990; Papageorgiou 1996; Campbell et al. 1998). This state is characterized by an increase of the effective antenna size of PSI. Exposing dark-adapted cells to light drives a state 2 to state 1 transition, typically within 10-30 s (Jallet et al. 2012; Kirilovsky 2007; Kaňa et al. 2009, 2012). State 1 is characterized by an increase in the effective antenna size of PSII, and hence, state 1 has a higher fluorescence yield (Kirilovsky et al. 2014). The cell is able to reversibly transition between state 1 and state 2 (state transitions) depending on the light conditions, in an effort to re-distribute excitation energy between PSI and PSII. See Mullineaux and EmlynJones (2005) for an in-depth review on state transitions.

This fluorescence study is an effort in disentangling these heavily intertwined energy and electron transfer pathways. After exciting PB with $590 \mathrm{~nm}$ light, spectrally resolved fluorescence traces were recorded over several minutes with $100 \mathrm{~ms}$ time resolution. The following mutants of Synechocystis were used in comparison to the wild-type (WT): (i) a PSI-lacking mutant ( $\triangle$ PSI hereafter); and (ii) a mutant without NDH-1 (M55 hereafter). In the absence of PSI, the cell lacks linear electron flow and, consequently, cannot produce $\mathrm{NADPH}$, thus requiring glucose to enable growth (Vermaas et al. 1994). It also presumably possesses additional photoprotective mechanisms related to overexpression of the high-light inducible protein (HliP) HliD (Sinha et al. 2012; Chidgey et al. 2014). By definition, state transitions do not take place in $\triangle$ PSI. The M55 mutant, lacking the $n d h B$ gene, does not assemble the NDH-1 complexes (Ogawa 1991). 
This is a highly disruptive mutation because these complexes have been reported to be involved in both respiratory and photosynthetic activities (Mi et al. 1995; Schmetterer 1994). Of particular interest is the disruption of respiratory activity in this mutant which causes a decreased input of electrons into the PQ pool in the dark. Since NDH-1 serves as an electron acceptor for ferredoxin $(\mathrm{Fd})$, it is also involved in CEF, which helps increase the PMF, leading to increased synthesis of ATP. The M55 mutant retains the capacity to undergo state transitions (Schuurmans et al. 2015). Furthermore, this mutant reportedly has impaired $\mathrm{CO}_{2}$ uptake (Ohkawa et al. 2000). In addition to the genetic disruptions, some samples have been treated with dichlorophenyl-dimethylurea (DCMU), a common inhibitor known for closing the PSII RCs as it blocks electron transfer from $Q_{\mathrm{A}}$ to $Q_{\mathrm{B}}$.

In an effort to anticipate from which pigment-protein complexes the fluorescence signal will originate we present in Fig. 1 a cartoon overview of putative fluorescent species that may play a role in the experiments reported in "Results" section. Beyond the evident species, i.e., the PB-PSII complex, a functional PB-PSII-PSI megacomplex (Fig. 1a, e, g) and the CpcG2-type of PB (Fig. 1b) are presented. The PB-PSII-PSI megacomplex has been isolated by Liu et al. (2013) and Kondo et al. (2009), and Gao et al. (2016) have reported on the $\mathrm{CpcG} 2$ type of PB. Chukhutsina et al. (2015) claim to observe such antenna in ultra-fast time-resolved fluorescence experiments. Additionally, we explore the possibility of a PB-PSII dimer complex with a single closed $\mathrm{RC}$ while the other remains open (see Fig. 1d, f).

\section{Materials and methods}

\section{Cell cultures}

Wild-type Synechocystis was obtained from D. Bhaya (Stanford). Wild-type cells were grown in a photobioreactor in turbidostat mode (growth rate ca. $0.05 \mathrm{~h}^{-1}$ ) at an optical density at $730 \mathrm{~nm}\left(\mathrm{OD}_{730}\right)$ of $0.40 \pm 0.01$, inoculated in modified BG-11 supplemented with $10 \mathrm{mM} \mathrm{NaHCO}$ and bubbled with $1 \%$ (v/v) $\mathrm{CO}_{2}$ in $\mathrm{N}_{2}$. The culture was illuminated with $636 \mathrm{~nm}$ light intensity of $80 \mu \mathrm{mol}$ photons $\mathrm{m}^{-2} \mathrm{~s}^{-1}$ as measured outside the reactor, opposite and centered relative to the light panel.

The PSI-deficient mutant of Synechocystis (Shen et al. 1993) was obtained from C. Funk (Umeå University, Sweden). Prior to preparing a liquid culture, cells were streaked on BG-11 agar plates from a stock stored at $-80{ }^{\circ} \mathrm{C}$ in $15 \%$ glycerol as described before (Acuña et al. 2018a). Subsequently, the plates were placed in an incubator (Versatile Environmental Test Chamber MLR-350H, Sanyo) at $30{ }^{\circ} \mathrm{C}$ with a humidified atmosphere with increased $\mathrm{CO}_{2}(2 \% \mathrm{v} / \mathrm{v})$. The plates were covered with layers of paper to reduce incident light intensity to below $5 \mu \mathrm{mol}$ photons $\mathrm{m}^{-2} \mathrm{~s}^{-1}$. For
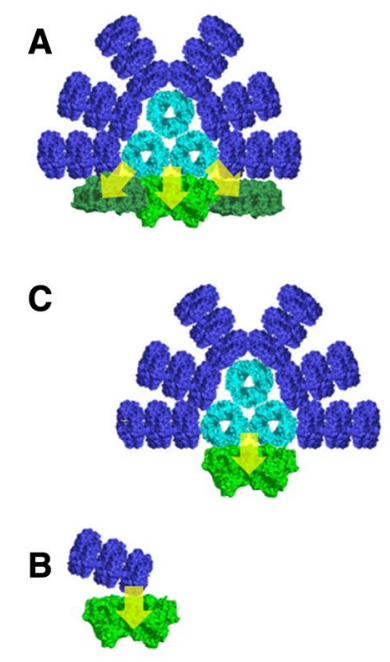
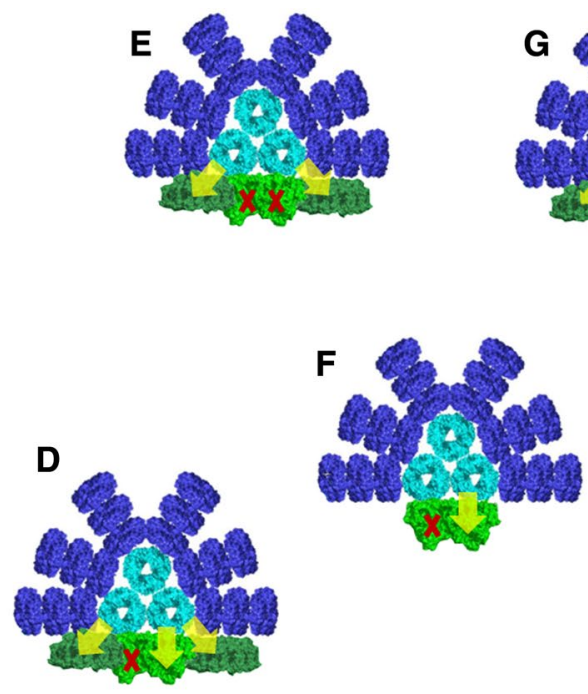
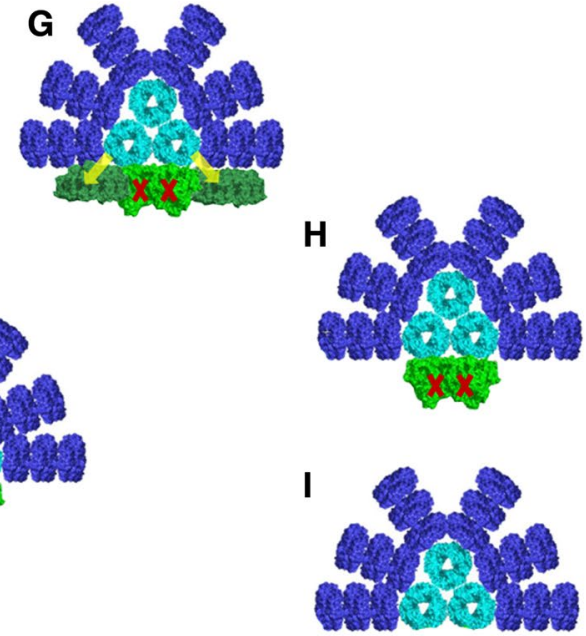

Fig. 1 Visualization of putative fluorescent species in Synechocystis ordered along the scale of their expected fluorescence quantum yield $\phi_{f}$. Yellow arrows represent EET from the PB core to PS I or II, their width indicates how efficient EET is from the PB to the respective PS. An " $X$ " stands for a closed PSII RC. a PB-PSII-PSI megacomplex efficiently quenched by both PSI I and II (open RC); b CpcG2 type of PB transferring energy to PSII; c PB-PSII complex with open
RCs, $\mathbf{d}$ PB-PSII-PSI megacomplex with a single RC closed and efficiently quenched by PSI and PSII; e PB-PSII-PSI megacomplex with fast energy transfer to PSI and closed PSII RCs; f PB-PSII complex with a single closed RC; g PB-PSII-PSI megacomplex with slow energy transfer to PSI and closed PSII RCs; h PB-PSII complex with closed RCs; i functionally uncoupled PB 
liquid cultures, $25 \mathrm{~mL}$ modified BG-11 (BG-11-PC, van Alphen et al. manuscript in preparation, substituting $\mathrm{NaNO}_{3}$ for $10 \mathrm{mM} \mathrm{NH}_{4} \mathrm{Cl}$ ) was inoculated and supplemented with $10 \mathrm{mM}$ glucose, $5 \mathrm{\mu g}^{-1} \mathrm{~mL}^{-1}$ chloramphenicol, and $25 \mathrm{mM}$ 1,4-piperazinedipropanesulfonic acid (PIPPS)-KOH buffered at pH 8.0 in a $100 \mathrm{~mL}$ flask (FB33131, Fisherbrand). In similar fashion to the agar plates, flasks were wrapped in layers of paper to reduce the incident light intensity to below $5 \mu \mathrm{mol}$ photons $\mathrm{m}^{-2} \mathrm{~s}^{-1}$. The flasks were placed in a shaking incubator (Innova 44, New Brunswick Scientific) at $120 \mathrm{rpm}$ and $30{ }^{\circ} \mathrm{C}$, equipped with a custom LED panel containing orange-red and blue LEDs of 632 and $451 \mathrm{~nm}$ (blue, both $8 \mathrm{~nm}$ full width at half maximum), respectively.

The M55 mutant was a gift from T. Ogawa (Nagoya University, Japan) and was grown in a chemostat kept at $30^{\circ} \mathrm{C}$, with a dilution rate of $D=0.03 \mathrm{~h}^{-1}$, illuminated by $635 \mathrm{~nm}$ light intensity of $40 \mu \mathrm{mol}$ photons $\mathrm{m}^{-2} \mathrm{~s}^{-1}$ and bubbled with $1 \%$ (v/v) $\mathrm{CO}_{2}$ in $\mathrm{N}_{2}$.

The abundance of Synechocystis sp. PCC6803 'wild types' provides an additional dimension of complexity, as there may be significant differences that are not readily apparent in routine culturing (Zavřel et al. 2017). We have attempted to account for these differences by subjecting the different wild-type strains to similar conditions as the derived mutant strains since introducing all mutations in a single wild-type strain was not successful. However, no spectral difference was observed between the 'Stanford' and 'Vermaas' wild-types in either photoautotrophic conditions as used for WT and M55, or heterotrophic conditions as used for the PSI-deficient mutant. All strains used, i.e., WT, $\mathrm{M} 55$, and $\Delta \mathrm{PSI}$, derive from the same branch of glucosetolerant, non-motile Synechocystis strains, i.e., the ATCC branch (Trautmann et al. 2012; Zavřel et al. 2017).

\section{Spectrally resolved fluorescence induction}

The multiple LED set-up described in Lambrev et al. (2010 and Acuña et al. (2016) was placed in the vicinity of where the Synechocystis cells were grown, ensuring that the cells were in well-defined physiological conditions with minimal time between sampling and measuring. For every strain, the illumination protocols (Fig. S1) have been applied to whole cells harvested from the same batch, diluted to an $\mathrm{OD}_{730}$ of 0.4 in fresh medium. In all cases, the background light $(590 \mathrm{~nm})$ intensity was $450 \mu \mathrm{mol}$ photons $\mathrm{m}^{-2} \mathrm{~s}^{-1}$ except during the saturating pulses where it was $1300 \mu \mathrm{mol}$ photons $\mathrm{m}^{-2} \mathrm{~s}^{-1}$. Each pulse (1 s duration) is analyzed with 10 fluorescence emission data points with a time resolution of $100 \mathrm{~ms}$. Cells have undergone varying DA times after harvesting prior to application of the illumination protocol (Fig. S1).

As reported previously (Acuña et al. 2018a), the last saturation pulse is applied in darkness to test the reversibility of the signal and to ensure that we can rule out any lightinduced damage, which we can for all the results reported here.

In experiments where DCMU was used, DCMU $(10 \mu \mathrm{M})$ was added to the sample before the experiment was conducted. It is well established that this concentration completely blocks the $\mathrm{Q}_{\mathrm{A}} \rightarrow \mathrm{Q}_{\mathrm{B}}$ electron transfer see e.g., Kaňa et al. (2012) and Kirilovsky et al. (1990).

\section{Additional elements of data analysis}

Here, we describe an extension of the spectral decomposition methods that have been described in Acuña et al. (2016). Acquired fluorescence spectra ( $p$ wavelengths) at $m$ time points can be represented by an $(m \times p)$ data matrix $\boldsymbol{\Psi}$. The Singular Value Decomposition (SVD) procedure (Golub and Van Loan 1996) decomposes $\boldsymbol{\Psi}$ according to

$\Psi=U \cdot S \cdot V^{\mathrm{T}}$

Into an $(m \times m)$ matrix $\boldsymbol{U}$, where the $m$ columns are called the left singular vectors $(l s v)$; the $(m \times p)$ diagonal matrix $S$ whose diagonal elements $\left(s_{1}, s_{2}, s_{3} \ldots\right)$ are called the singular values and the transpose of the $(p \times p)$ matrix $\boldsymbol{V}, \boldsymbol{V}^{\mathbf{T}}$, where the $p$ rows are called the right singular vectors $(r s v)$. Timedependent characteristics are contained in the $l s v$, while the $r s v$ are a linear combination of the species associated spectra (SAS). The original matrix $\boldsymbol{\Psi}$ can be satisfactorily reconstructed by means of the $n$ most significant singular vectors yielding a (nt $\times \mathrm{n} \lambda)$ matrix $\boldsymbol{\Psi}_{\mathrm{n}}$. In Acuña et al. (2016) data matrices of rank $n=2$ were analyzed. While the core of the method remains the same, we also present data matrices that are of rank $n=3$, meaning that the transformation matrix applied to the singular vectors had to be correspondingly expanded.

We seek a mathematical transformation $\Psi_{\mathrm{n}}=U \cdot S \cdot\left(A^{-1} \cdot A\right) \cdot V^{\mathrm{T}}$ to resolve the SAS and their timedependent concentrations. For a rank $n=3$ matrix, $A$ has the following form:

$A=\left(\begin{array}{ccc}1 & a_{12} & a_{13} \\ a_{21} & a_{22} & a_{23} \\ a_{31} & a_{32} & a_{33}\end{array}\right)$

and the final decomposition of $\boldsymbol{\Psi}_{(\mathrm{n}=3)}$ is expressed as a linear combination of three $n t \times 1$ vectors corresponding to the concentrations and the $1 \times \mathrm{n} \lambda$ vectors corresponding to the $\mathrm{SAS}_{\mathrm{i} \lambda}$ :

$\Psi_{\mathrm{n}=3}=c_{t 1} \mathrm{SAS}_{1 \lambda}+c_{t 2} \mathrm{SAS}_{2 \lambda}+c_{t 3} \mathrm{SAS}_{3 \lambda}$.

Hereafter, we will refer to these vectors using the simplified notation: $c_{1}, c_{2}, c_{3}, \mathrm{SAS}_{1}, \mathrm{SAS}_{2}$, and $\mathrm{SAS}_{3}$. Furthermore, a number of criteria are followed to judge whether these transformed vectors are biophysically meaningful. 
With the expansion to a rank $n=3$ matrix, the number of parameters to be estimated increases; this is why, additionally to the criteria presented in our previous work (Acuña et al. 2016), we have added, for each SAS, SAS smoothness-penalties:

penalty $_{\text {smoothness }}=\sum_{j} w_{j} \cdot\left|\int \frac{\partial^{2}}{\partial \lambda^{2}} S A S_{j}(\lambda)\right|$.

This is one among other contributions to the objective function that we seek to minimize. See the SI for the explicit objective function used in this work.

Finally, each concentration value $c_{\mathrm{ti}}$ output at the position $t$ that corresponds to a saturating pulse is multiplied by a parameter $p_{\mathrm{f}}$ that accounts for the difference in light intensity between the background illumination and the saturating pulse. Thus, relative concentrations that are independent of the light intensity are estimated.

\section{Results}

In the first section, we discuss results obtained following different protocols. First, we show results of the photosynthetically "simpler" system, $\Delta$ PSI (which does not contain any of the species depicted in Fig. 1a, d, e, g) after a series of DA periods. Then, we move on to the PSI-containing samples of the strains M55 and WT, first showing results of DCMUtreated samples with two different DA periods, then, a series of several DA periods with intact cells (without DCMU).

\section{$\Delta$ PSI cells}

We report analyses of fluorescence data acquired from $\Delta$ PSI cells that have undergone different dark-adaptation periods. The rank of the data matrices for all DA periods is at least three (one example is shown in Fig. S2). A decomposition analysis using the main three components has been performed. In Fig. 2, we show results for the two DA periods 1 and $34 \mathrm{~min}$. The full series of six DA periods is shown in Fig. S3. Based on visual inspection of the F660/F680 ratio

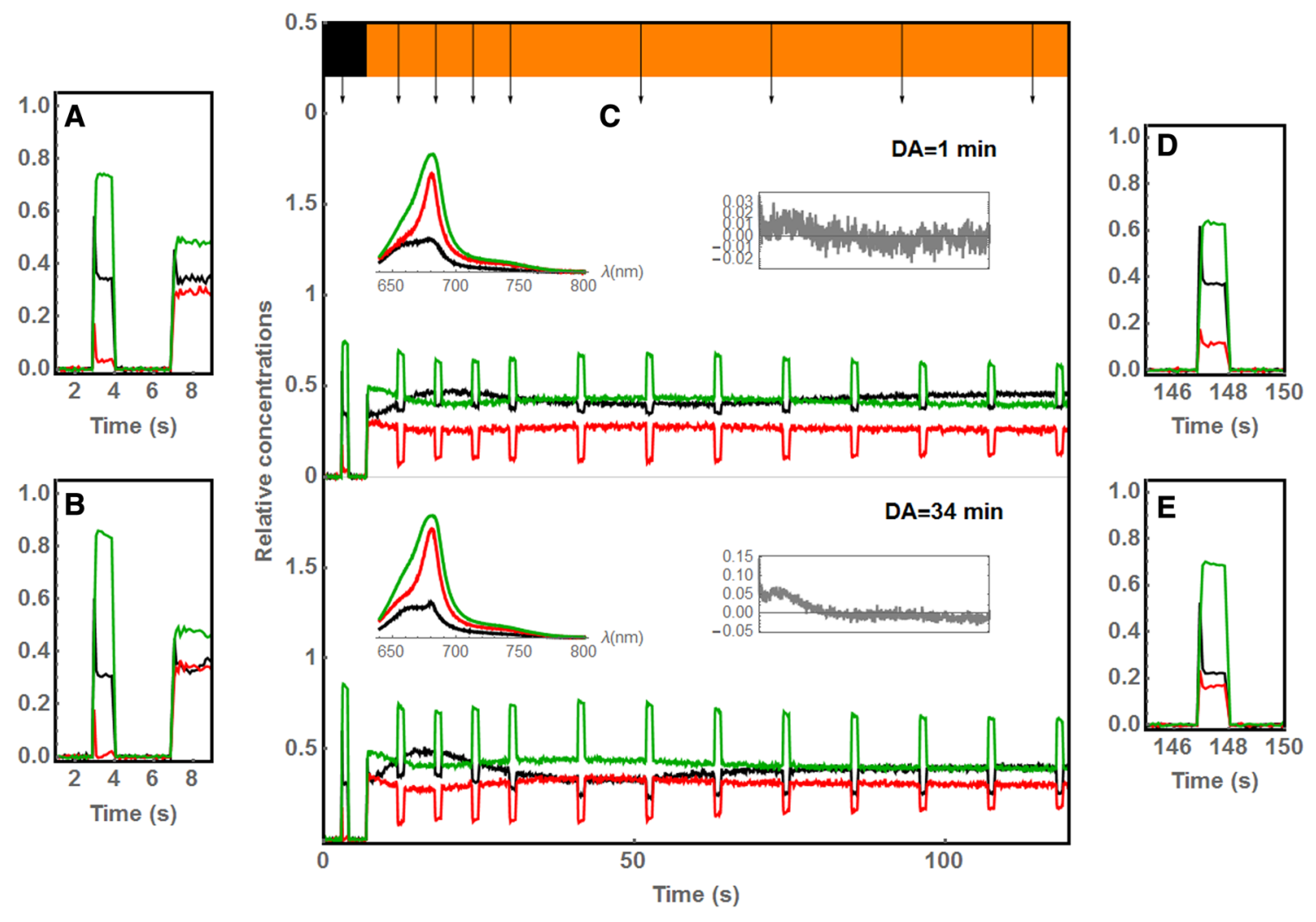

Fig. 2 Spectral decomposition of two independent experiments carried out on $\triangle$ PSI cells. $\mathbf{c}$ shows the first 2 min of the light protocol for the two dark-adaptation (DA) periods 1 and $34 \mathrm{~min}$. For each condition, a zoom view of the first (last) pulse is depicted in $\mathbf{a}$ and $\mathbf{b}$ (d and $\mathbf{e}$ ). The corresponding SASs (shown as insets) are depicted on $\mathbf{c}$.
The colored bar on top illustrates the light regime: $450 \mu$ mol photons $\mathrm{m}^{-2} \mathrm{~s}^{-1}$ of $590 \mathrm{~nm}$ light (orange) or darkness (black). In gray, the deviation in the sum of concentrations from unity. For the full data matrices and the complete series of DA periods, see Fig. S3 
and the relative amplitudes (fluorescence quantum yield) of the SASs, they are interpreted as follows: $\mathrm{SAS}_{3}$ (green) as a PB-PSII complex with fully closed RCs ( -closed) (Fig. 1h); $\mathrm{SAS}_{2}$ (red) as a PB-PSII with partially closed PSII RCs ( $p$-closed) (Fig. 1f) and, SAS $_{1}$ (black) as a PB-PSII complex with PSII RCs open (Fig. 1c) or PSII quenched by HliP (Acuña et al. 2016).

Most probably the SAS of a PB-PSII complex with PSII RCs open or quenched by HliP differ slightly, leading to a data matrix of rank 4 (see Fig. S2). Since the analysis is performed using the main three components, it is not possible to describe the data matrix fully. This manifests in the sum of concentrations shown in gray on the insets of Fig. 2c. For short DA periods, the sum of concentrations is close to flat. As DA increases, however, it becomes increasingly difficult to obtain a fully flat pattern, especially during initial phases. Also, while $\mathrm{SAS}_{2}$ and $\mathrm{SAS}_{3}$ give robust results, $\mathrm{SAS}_{1}$ because of its low $\phi_{\mathrm{f}}$ provides the highest uncertainty. Even though a quenched component is consistently required to minimize the residuals, determining the exact shape and the F660/F680 ratio becomes non-trivial due to only subtle changes in the sum of concentrations profile. Certainly, the optimization routine may be compensating for the lack of the fourth component, in which case $\mathrm{SAS}_{1}$ is expected to be a mixture of the two quenched components. Assuming so, this would explain that the $c_{1}$ (black) decreases during the saturating pulses, as expected for open complexes (Fig. 1c), while tending towards a baseline level of around $40 \%$ (cf. Fig. 2, Table S2). These results suggest the existence of two quenched components of similar SAS, one of which is photochemically, the other non-photochemically quenched. Judging from the deviations in the sum of concentrations, the photochemically quenched component plays a role predominantly during the first $30 \mathrm{~s}$.

The rationale behind the $p$-closure interpretation $\left(\mathrm{SAS}_{2}\right)$ lies in the decreasing $c_{2}$ (red) during the saturating pulses, as opposed to the rising $c_{3}$ (green), which is formed as more light is shone onto the sample. Indeed, despite the strong $\mathrm{Chl}$ $a$ signature of $\mathrm{SAS}_{2}$, the organism demonstrates the ability to close more RCs as $c_{2}$ decreases. Indeed, $c_{2}$ behaves as a partially 'open' species. We will discuss some possible origins of the $p$-closed RCs in the "Discussion" section.

As for the concentration profiles shown in Fig. 2c, it is interesting to note that the onset of the background illumination triggers a slow (several seconds) population exchange, predominantly between species $\mathrm{SAS}_{1}$ and $\mathrm{SAS}_{3}$ while $\mathrm{SAS}_{2}$ remains fairly flat. Relatively constant fluorescence levels are observed for times $t>40 \mathrm{~s}$. Furthermore, contrary to previous observations in rank 2 analyses of $\Delta$ PSI data (Acuña et al. 2018a), the concentration profiles show no systematic changes which may be unequivocally related to the DA periods. This is probably due to a crucial difference in the growth conditions of the different batches: while in Acuña et al. (2018a) $\mathrm{NaNO}_{3}$ was used as the nitrogen source in the medium, here we have used $\mathrm{NH}_{4} \mathrm{Cl}$. The former may result in a deficiency in nitrogen assimilation, as the reduction of $\mathrm{NO}_{3}{ }^{-}$to $\mathrm{NH}_{3}$ requires the direct output of PSI, i.e., reduced ferredoxin. Thus, in the absence of PSI, this reaction is hampered. Additionally, $\mathrm{NH}_{4} \mathrm{Cl}$ has a lower 'electron cost' than $\mathrm{NaNO}_{3}$ since $\mathrm{NH}_{4}{ }^{+}$can be directly used for metabolic reactions unlike $\mathrm{NO}_{3}{ }^{-}$which has to be reduced to $\mathrm{NH}_{3}$ first, requiring a total of eight electrons. This may lead to less of an overload in the electron transfer pathways of this mutant and, as a result, the cell may need to rely on less regulatory mechanisms during darkness explaining why different DA periods do not result in the systematic patterns observed by Acuña et al. (2018a). Nevertheless, the response is highly dynamic with none of the three concentration profiles standing out for being the predominant one.

\section{M55 and WT cells pre-treated with DCMU}

The data matrices of experiments carried out on WT and M55 cells are of rank 3. The cells were pre-treated with DCMU and underwent two different DA periods as shown in Fig. 3i, j. The three SASs are shown in the insets. A quenched species, SAS $_{1}$ (black), is present in all samples. The interpretation of $\mathrm{SAS}_{3}$ (green) is a PB-PSII-PSI complex with $f$-closed RCs. Here again, $\mathrm{SAS}_{2}$ (red) has a quenched $660 \mathrm{~nm}$ emission relative to $\mathrm{SAS}_{3}$ and this is interpreted as a PB-PSII-PSI complex with $p$-closed RCs. Saturating pulses are still able to close a certain fraction of PSII RCs as manifested in the decrease of both $c_{1}$ and $c_{2}$. Interestingly, the fraction that can be closed in M55 is smaller than in WT (cf. $F_{\mathrm{S}}$ values in Table S1), suggesting that a fully assembled NDH-1 unit (in the presence of DCMU) contributes to efficiently alleviating the excitation pressure on PSII. As for the time profiles, we read the following: for 8-min dark-adapted samples, $c_{1}$ shows a slight increase suggesting that formation of the quenched species (black) is favored in darkness. This is valid for both M55 (cf. Fig. 3a, c) and WT (cf. Fig. 3e, g). Also visible on these panels is the fact that WT forms more of the quenched species as compared to M55. Note that $c_{1}<c_{2}$ systematically for M55 as background light sets in. This ratio is reversed for the WT. Moreover, while M55 very quickly finds a steady-state level that varies little over time, WT shows an equilibration phase of ca. $10 \mathrm{~s}$ during which the quenched species decreases and then levels off.

\section{M55 cells without DCMU}

Fluorescence signals from M55 cells that have undergone DA periods of 1 and 34 min are shown in Fig. 4. The full DA series of analyses of M55 cells is shown in Fig. S4, with a zoom-view of the first $35 \mathrm{~s}$ in Fig. S5. The SASs 

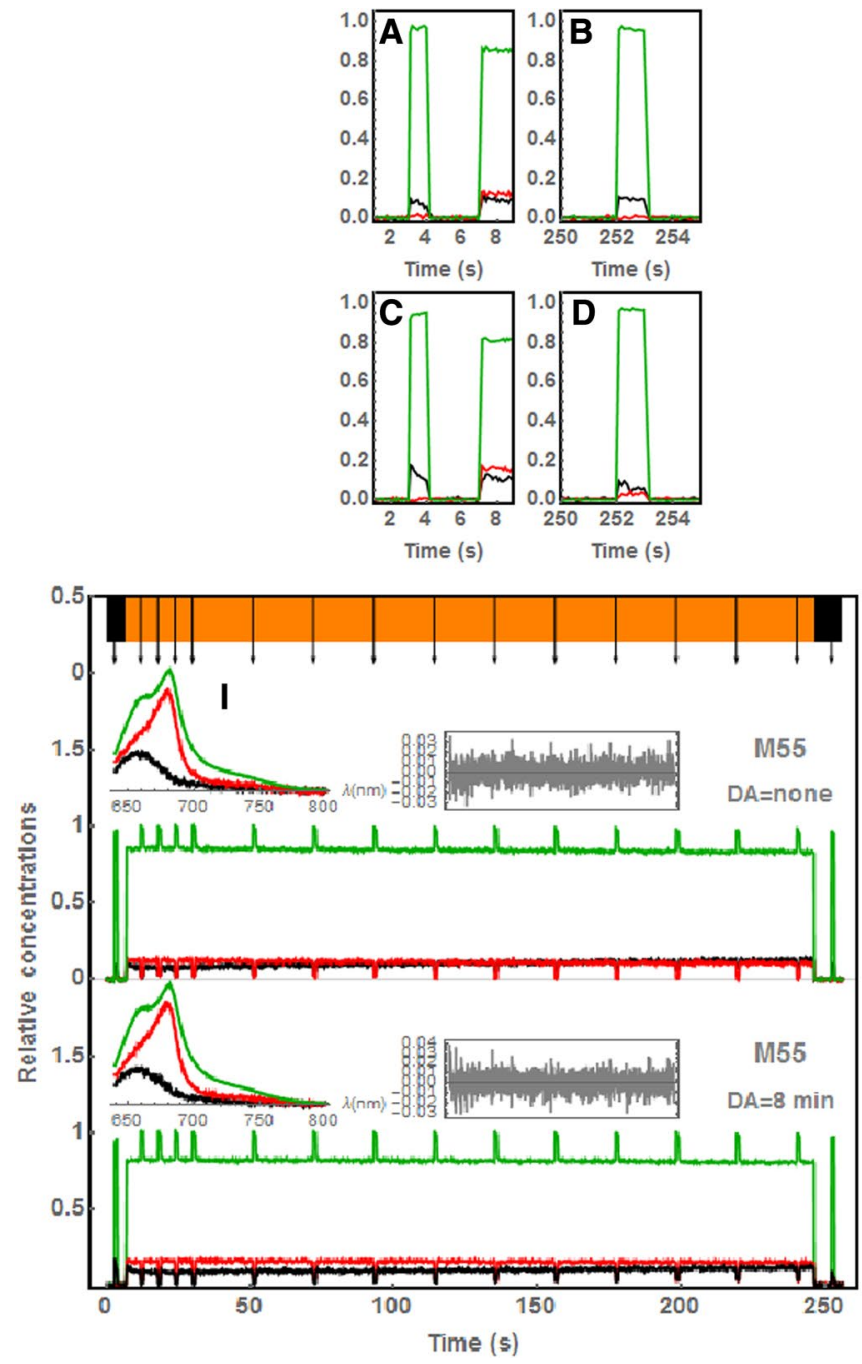

Fig. 3 Spectral decomposition of two independent experiments with two distinct dark-adaptation (DA) periods carried out on M55 (left) and WT (right) cells with DCMU. The $\mathbf{a}$ and $\mathbf{b}$ depict a zoom view into the first and last pulse of M55 with DA none; c and d M55, $\mathrm{DA}=8 \mathrm{~min} ; \mathbf{e}$ and $\mathbf{f} \mathrm{WT}, \mathrm{DA}=$ none; $\mathbf{g}$ and $\mathbf{h} \mathrm{WT}, \mathrm{DA}=8 \mathrm{~min}$. The

are interpreted as follows: $\mathrm{SAS}_{3}$ (green) as a PB-PSII-PSI complex with moderate EET from PB to PSI and $f$-closed PSII RCs (see Fig. 1g), SAS ${ }_{2}$ (red) as a PB-PSII-PSI megacomplex with fast energy transfer to PSI and with $p$-closed RCs (see Fig. 1e), and, SAS 1 (black) as a PB-PSII(-PSI) complex with $f$-open PSII RCs (see Fig. 1a). EET from PB to PSI most probably occurs via ApcD (Dong et al. 2009) and depending on whether it is slow or fast, more or less $680 \mathrm{~nm}$ light is able to escape. This would explain the strong decrease in the F660/F680 ratio, which serves as a proxy for how much excitation light was ultimately trapped by PSI. The corresponding concentration profiles also show interesting dynamics: unlike the DCMU-treated samples, the shape of the pulses resembles much less that of a step function (cf. Figs. 3a, 4a); take for instance $c_{3}$ which after reaching a
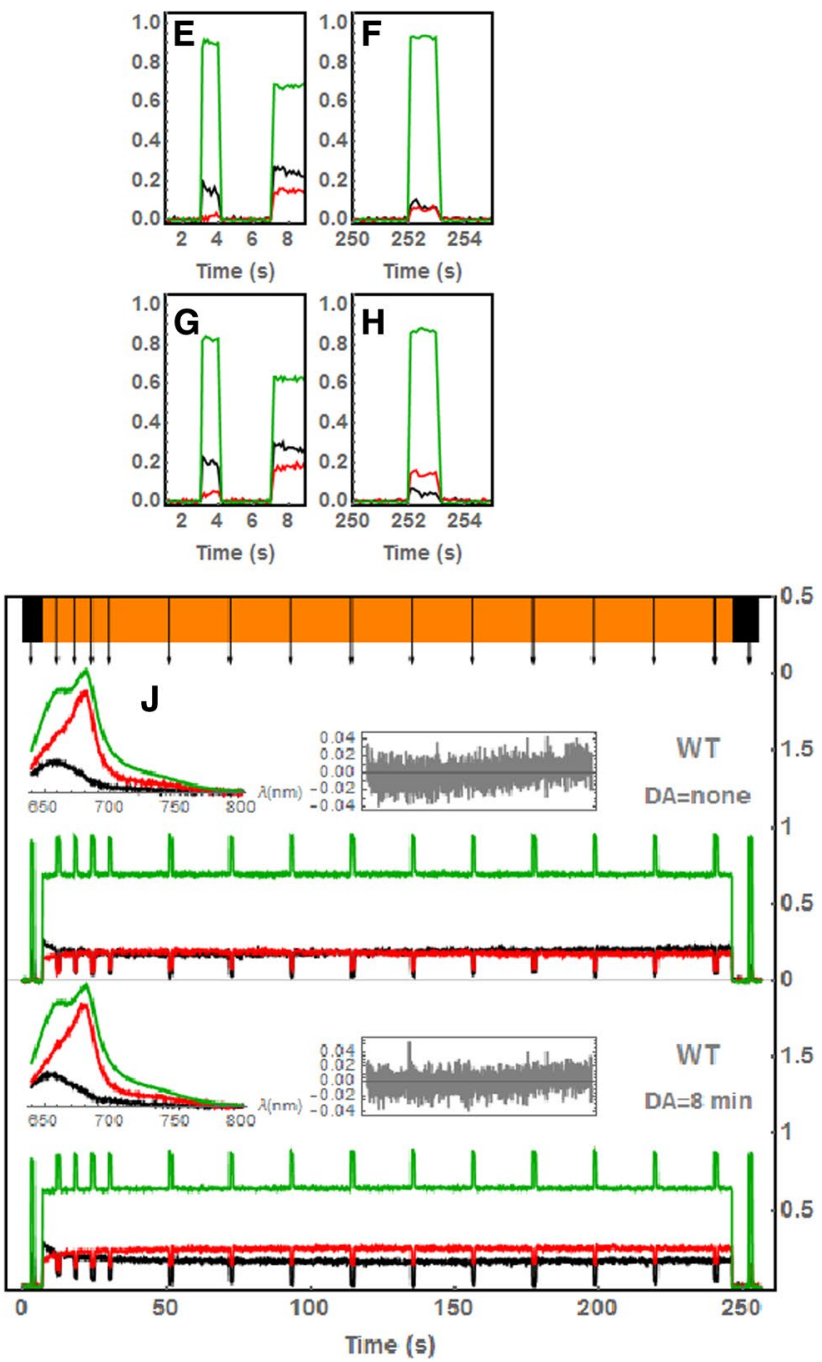

$\mathbf{i}$ and $\mathbf{j}$ show the full time profiles. The corresponding SASs and the deviation in the sum of concentrations from unity are shown as insets. The colored bar on top illustrates the light regime: $450 \mu \mathrm{mol}$ photons $\mathrm{m}^{-2} \mathrm{~s}^{-1}$ of $590 \mathrm{~nm}$ light (orange) or darkness (black)

maximum within the first $200-300 \mathrm{~ms}$, decreases as much as $10 \%$ within the duration of the pulse. This suggests efficient electron transfer from PSII to the PQ pool and subsequent re-opening of PSII RCs. The behavior of $c_{2}$ is of particular interest: while for short DA periods, during the first pulse, $c_{2}$ tends towards zero. For DA $=8 \mathrm{~min}, c_{2}$ averages to $5 \%$ and it increases to 14 and $19 \%$ for DA of 21 and $34 \mathrm{~min}$, respectively (see Table $\mathrm{S} 2$ ). Hence, darkness favors the formation of the species $\mathrm{SAS}_{2}$. Seemingly, during the first $20 \mathrm{~s}$ of background light this occurs at the expense of the species $\mathrm{SAS}_{3}$ only. Thus, for longer DA periods, $c_{2}\left(c_{3}\right)$ has a larger (smaller) initial concentration and, as background light sets in, it continues to gradually increase (decrease) during the first 10-20 s resulting, for long DA periods, in a momentarily larger concentration of $\mathrm{SAS}_{2}$ than $\mathrm{SAS}_{3}$. Then, $c_{3}$ tends to 

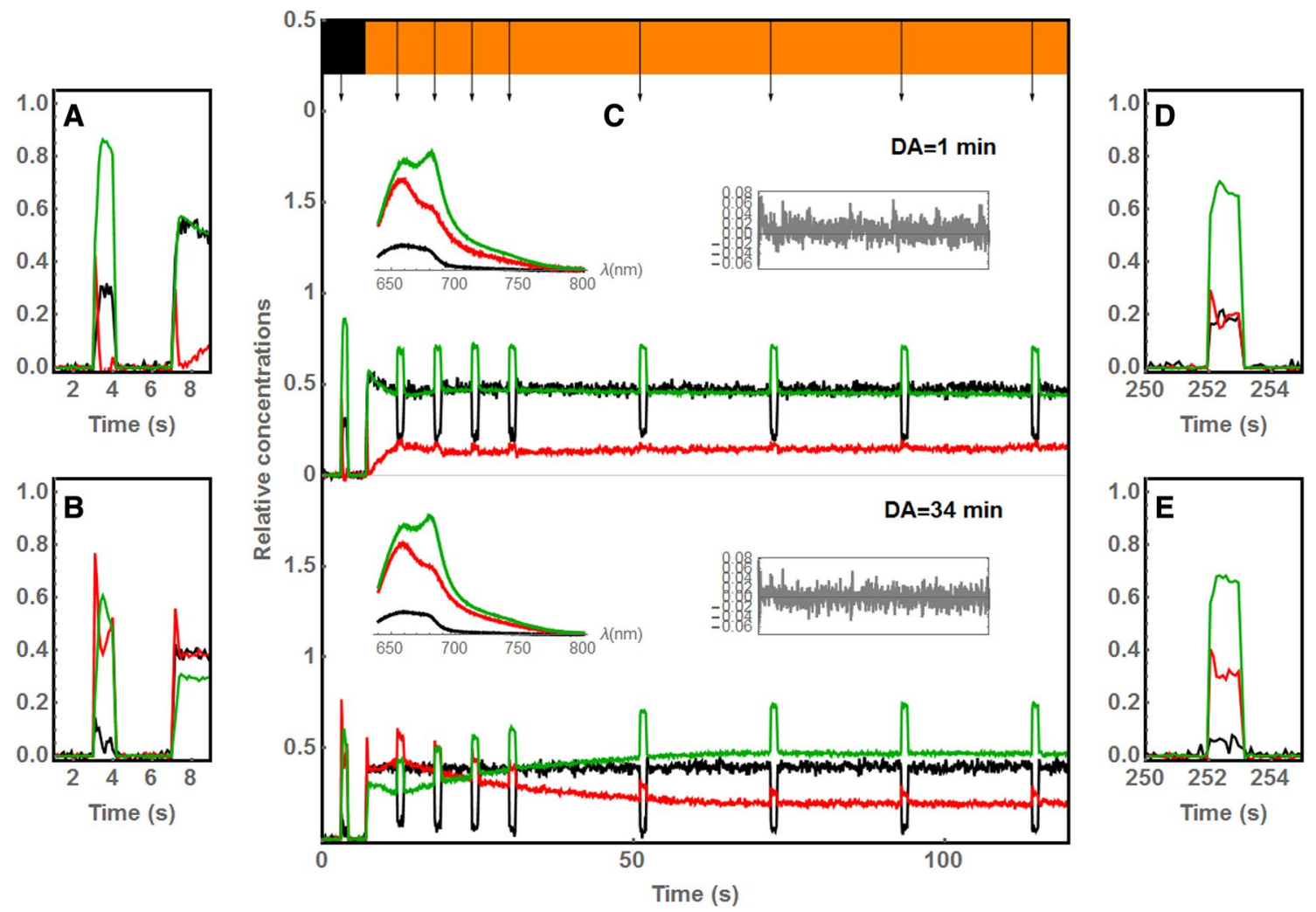

Fig. 4 Spectral decomposition of two independent experiments carried out on WT cells without DCMU. c shows the first 2 min of the light protocol for the two dark-adaptation (DA) periods 1 and $34 \mathrm{~min}$. For each condition, a zoom view of the first (last) pulse is depicted in a and $\mathbf{b}$ (d and e). The corresponding SASs (shown as

a $F_{\mathrm{S}}$ level of ca. $40 \%$ and it restores that level quite consistently regardless of the initial conditions. We attribute this behavior to a state 2 to state 1 transition. Furthermore, the $c_{1} F_{\mathrm{S}}$ value is also ca. $40 \%$ (see Table $\mathrm{S} 2$ ) in all experiments i.e., the background light is only able to close $60 \%$ of the PSII RCs. In both WT and M55, DA favors the formation of a megacomplex in which fast energy transfer from $\mathrm{PB}$ to PSI (see Fig. 1e) is ensured (Liu et al. 2013), consequently increasing its antenna size and ultimately affecting the ratio CEF/LEF.

\section{WT cells without DCMU}

Fluorescence signals from WT cells that have undergone the two different DA periods 1 and 34 min are shown in Fig. 5. The full DA series of analyses of WT cells is shown in Fig. S6, with a zoom-view of the first $35 \mathrm{~s}$ in Fig. S7. The SASs are interpreted as in the previous section. Also in this series of experiments, the initial $c_{2}$ (red) increases with longer DA periods, and, as visible from the first pulse of every experiment (see Fig. S6a-f), it reaches up to $40 \%$ for $\mathrm{DA}=34 \mathrm{~min}$. As background illumination sets in, the levels insets) are depicted on c. The colored bar on top illustrates the light regime: $450 \mu \mathrm{mol}$ photons $\mathrm{m}^{-2} \mathrm{~s}^{-1}$ of $590 \mathrm{~nm}$ light (orange) or darkness (black). In gray, the deviation in the sum of concentrations from unity. For the full data matrices and the complete series of DA periods, see Fig. S4

of open and closed complexes (black and green) are similar. For all experiments, the concentration $c_{1}$ i.e., the open complexes, also shows a relatively steady level throughout the whole period of background illumination only decreasing during saturating pulses. Similarly to the M55 mutant, the initial $c_{2}$ and $c_{3}$ depend on DA.

\section{Discussion}

\section{Partial closure of PSII RCs}

As there is no energetic interaction between PSII reaction centers (Mullineaux and Holzwarth 1993), we can assume that PSII fluorescence is directly proportional to the fraction of $Q_{\mathrm{A}}$; therefore, partial closure of PSII RCs means that the light pressure does not suffice to completely depopulate the PQ pool. The question is what is/are the underlying mechanism(s) that allow the PQ pool to cope with the excitation pressure during the background illumination phase?

One possibility is that a small fraction of PB-PSII-PSI complexes have one of the RCs of the PSII dimer closed 


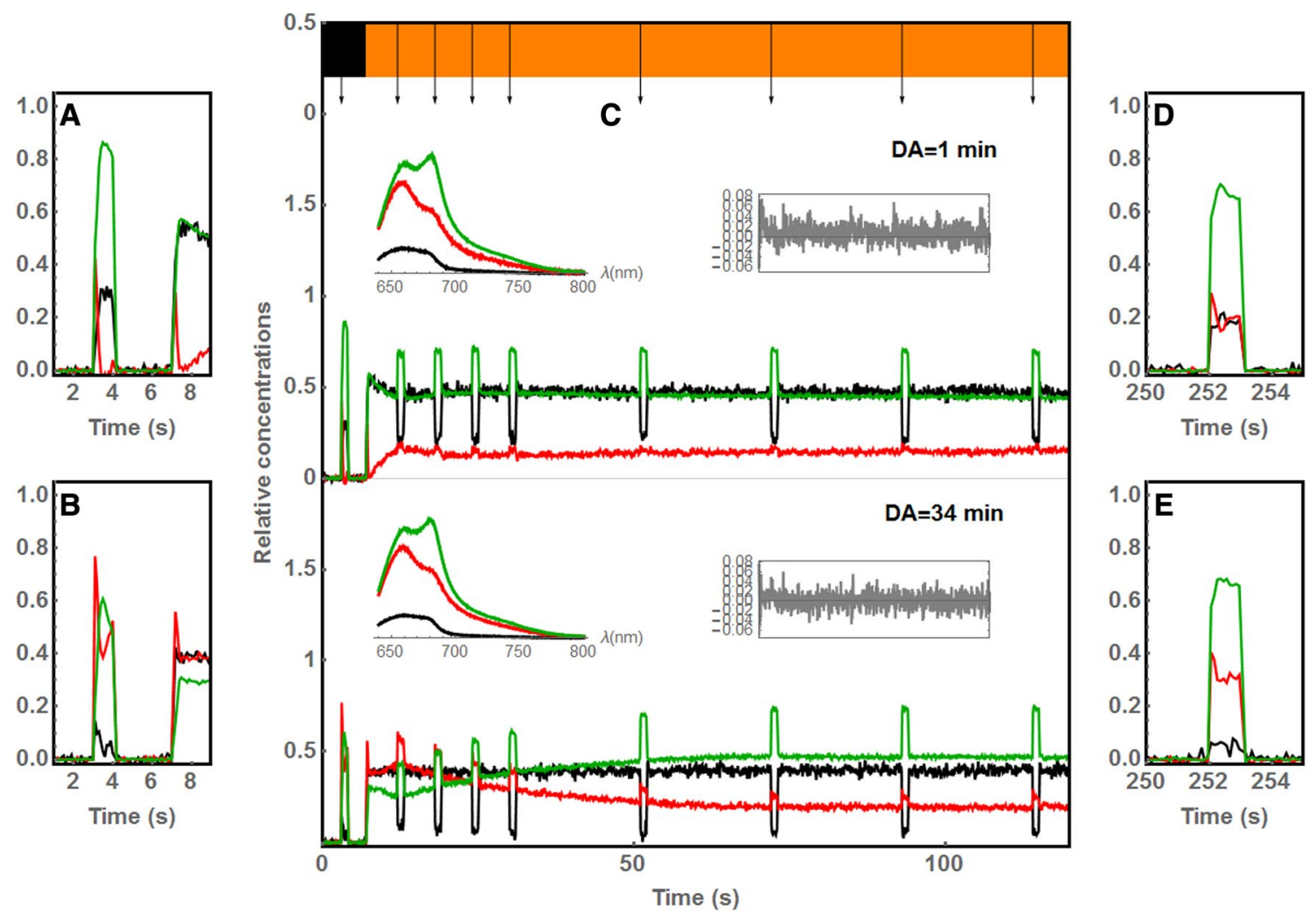

Fig. 5 Spectral decomposition of two independent experiments carried out on WT cells without DCMU. c shows the first 2 min of the light protocol for the two dark-adaptation (DA) periods 1 and $34 \mathrm{~min}$. For each condition, a zoom view of the first (last) pulse is depicted in $\mathbf{a}$ and $\mathbf{b}$ (d and e). The corresponding SASs (shown as

while the other remains open. In this case, the $p$-closed contribution, in the $\Delta$ PSI experiments, would be attributed to the species depicted in Fig. 1f; and to the species in Fig. 1d, for the PSI-containing samples.

A major conflict with this interpretation is provided by the DCMU experiments, where $p$-closure cannot be ascribed to PSII RCs that would have remained open. The capacity to keep PSII RCs open would have to stem from an additional pool electrons can escape to, for example, a pool of Flv2/4 proteins. Consequently, the very definition of 'open' would have to be extended; while hitherto 'open' exclusively referred to the PQ-pool ability to accept electrons from the PSII special pair, there would be two types of mechanisms that contribute to alleviating the excitation pressure on PSII RCs: $p q$-open when related to the capacity of the PQ-pool and $f v$-open when related to that of the Flv2/4 heterodimer. Thus, we hypothesize that DCMU ensures that all RCs are $p q$-closed, but that the alternative electron transport via the Flv2/4 pathway would however remain accessible.

Although the exact binding site and rate are still unknown for Flv2/4, the assumption in this case would have to be that $Q_{\mathrm{B}}$ is not involved in the electron transfer. Instead, we insets) are depicted on c. The colored bar on top illustrates the light regime: $450 \mu \mathrm{mol}$ photons $\mathrm{m}^{-2} \mathrm{~s}^{-1}$ of $590 \mathrm{~nm}$ light (orange) or darkness (black). In gray, the deviation in the sum of concentrations from unity. For the full data matrices and the complete series of DA periods, see Fig. S6

observe a quenched $660 \mathrm{~nm}$ emission which would align well with the observations by Bersanini et al. (2014) that point towards Flv2/4-related activity with concomitant PB co-operation. Indeed, even the properties of a PB "super core" as recently proposed by Zlenko et al. (2017) might be required. Furthermore, as manifested in the lower $F_{\mathrm{S}}$ levels of $c_{3}$ in WT (Table S1), the amount of $f l v$-closure is ca. $20 \%$ larger (for both DA periods) in the WT than it is in strain M55. This directly points at a role of NDH-1 in keeping the PQ-pool reduced (Chen 2017). Certainly, there must be additional acceptors to Flv2/4 that explain why, despite NDH-1 dysfunction, the RCs are not fully $f v$-closed in M55. Spectrally resolving the fluorescence signal of DCMU pretreated cells of a $\Delta f l v 2 / 4$ mutant as well as measurements of expression levels of these proteins will have to support such an interpretation and test this hypothesis.

\section{Time dependence in M55 and WT cells without DCMU}

Without DCMU i.e., with unblocked quinone acceptors, the F660/F680 of $\mathrm{SAS}_{2}$ greatly increases, evidencing the 
efficient re-opening of RCs. Also, then the time profiles, especially $c_{2}$ and $c_{3}$, are much more dynamic. Here, we lay the focus of the discussion on three different aspects: (i) the overall behavior of the $F_{\mathrm{S}}$ fluorescence levels, (ii) some observations specific to the $F_{\mathrm{M}}$ levels, and (iii) DA dependence of points (i) and (ii). To conclude, we will compare the results obtained with the M55 mutant and the WT.

Figures 6 and 7 show $c_{2}$ and $c_{3}$, respectively, during the first minute of the light protocol. In Fig. 6, the zoom view of $c_{2}$, which corresponds to the concentration of a PB-PSII-PSI

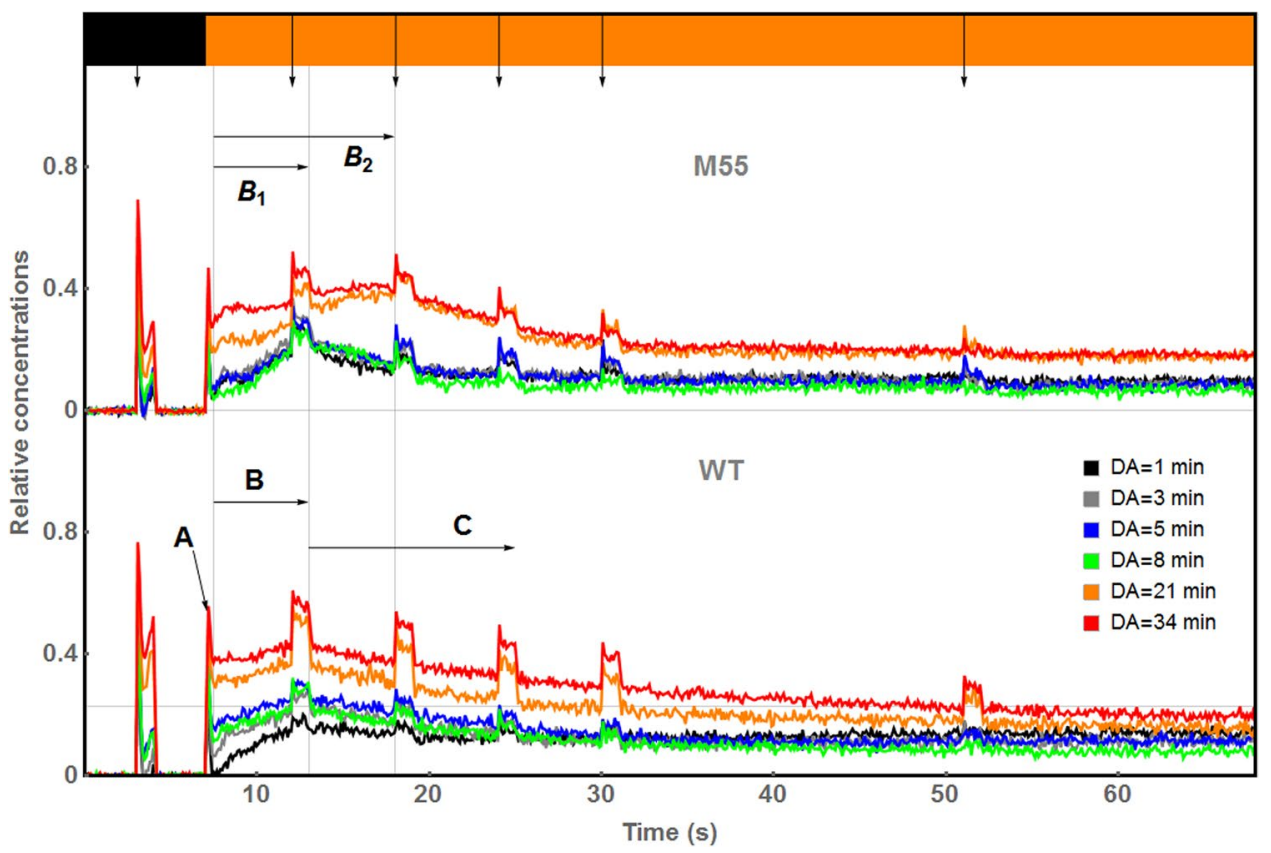

Fig. 6 Zoom-view into the first minute of the light protocol for WT and the M55 mutant. The profile $c_{2}$, corresponding to megacomplexes with fast PB to PSI energy transfer is shown for all DA periods. Background illumination sets in at $t=7 \mathrm{~s}$. Immediately afterwards, three intervals are indicated: a first $300 \mathrm{~ms}$ after onset of background illumination; b $7.3 \mathrm{~s}<t<13 \mathrm{~s}$ and $\mathbf{c} 13 \mathrm{~s}<t$. a is characterized by a rapid population increase and decrease presumably due to closure of a single RC followed by a population of $c_{3}$ (Fig. 7), $\mathbf{b}$ is characterized by a steady increase reaching a maximum and during $\mathbf{c}$ the profiles decline steadily again. In the case of M55 cells, DA times of 21 and 34 min display a longer period of steady increase, $B_{2}$, reaching a maximum value at $t=20 \mathrm{~s}$
Fig. 7 Zoom-view into the first minute of the light protocol for WT and the M55 mutant. The profile $c_{3}$, corresponding to megacomplexes with slow PB to PSI energy transfer, is shown for all DA periods. The decrease in the initial concentration values correlates with increasing DA periods. This is interpreted as a state 1 to state 2 transition. Background illumination triggers a state 2 to state 1 transition

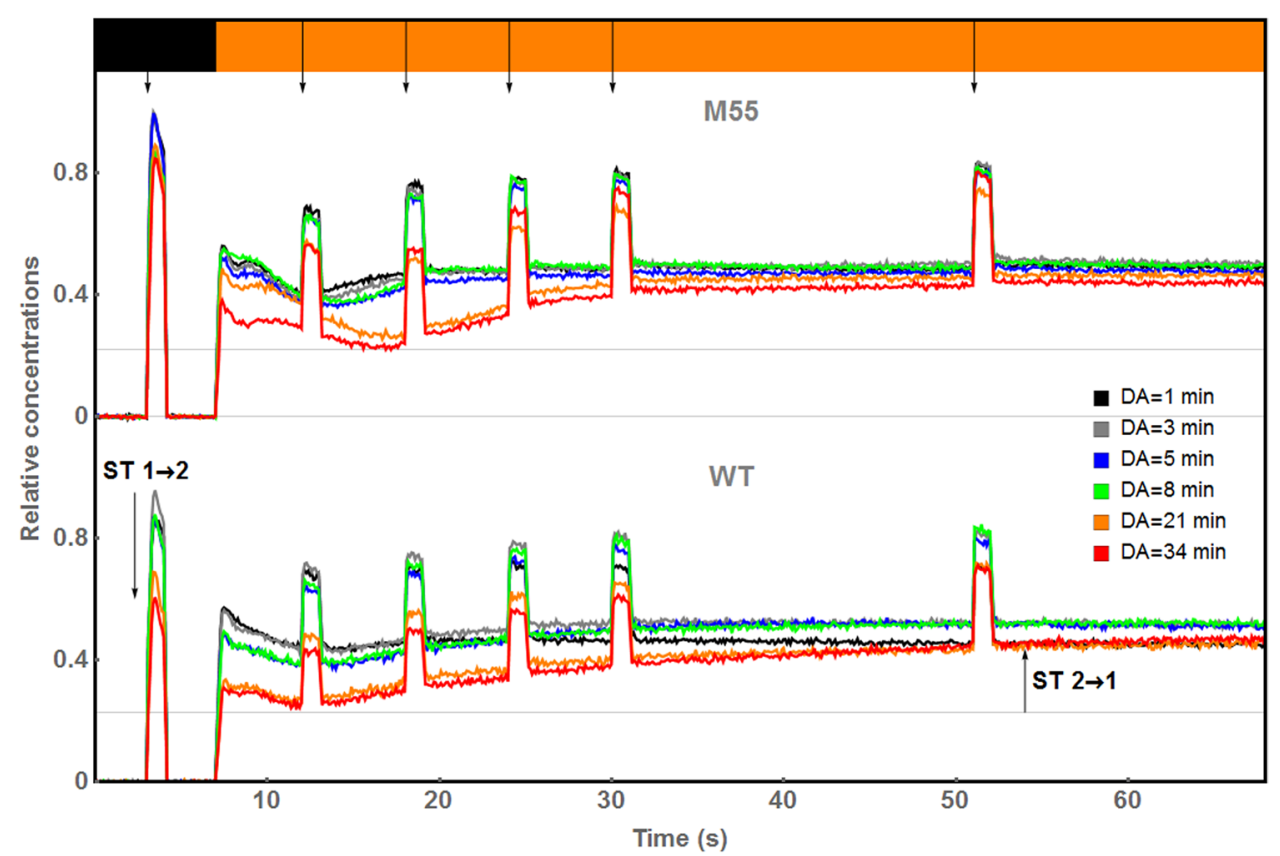


complex with fast energy transfer to PSI, reveals grosso modo three different phases of the $F_{\mathrm{S}}$ levels: as background illumination sets in, there is a quick equilibration that happens within $300 \mathrm{~ms}$ and that we refer to as phase A. During this phase, the $c_{2}$ profile quickly decreases until a minimum value is reached while $c_{3}$ shows a correlated increase, just as observed during the first data points of the saturating pulse applied in darkness (cf. Figs. S5 and S7). We conclude that this is due to opening/closing of RCs, meaning that $\mathrm{SAS}_{2}$ has a hybrid nature: the PB-PSII-PSI megacomplex with fast energy transfer to PSI and $p$-closed (Fig. 1e) gets to the $f$-closed state within these first $300 \mathrm{~ms}$ of illumination. Thus, we see both the complexes with open RCs being closed as a fast (within ms) decrease in $c_{2}$ and the steady $F_{\mathrm{S}}$ level (changing only in the scale of seconds) of the complexes with already closed RCs. The phase $\mathrm{B}(7.3<t<13 \mathrm{~s})$ is characterized by a progressive increase of the $F_{\mathrm{S}}$ level, which we interpret as the cell first adopting a configuration in which the energy transfer to PSI is increased, presumably to decrease the excitation pressure on PSII. After reaching a maximum, $c_{2}$ enters a phase $\mathrm{C}$ of steady decline attributed to a state 2 to state 1 transition, and which leads to an increase in the number of PB-PSII-PSI complexes with slow energy transfer to PSI (see Fig. 1g). Note, however, that while the phase B in the WT consistently lasts ca. $5 \mathrm{~s}$ for all DA periods, in M55, DA periods of 21 and 34 min result in a delayed state 2 to state 1 transition (see Fig. 6). For DA of 1, 3, 5, and $8 \mathrm{~min}$ the phase $B_{1}$ is as long as the phase $\mathrm{B}$ observed in WT, but for DA periods of 21 and 34 min (orange and red in Fig. 6), the phase $B_{2}$ extends until $t=18 \mathrm{~s}$. Seemingly, with a lacking NDH-1 unit, which impairs electron transfer into the PQ pool and CEF, the cell needs a longer time, during which the amount of megacomplexes with fast PB $\rightarrow$ PSI energy transfer further accumulates, before triggering a state 2 to state 1 transition.

Another instructive aspect of the time profiles is the behavior of the $F_{\mathrm{M}}$ levels during the $1000 \mathrm{~ms}(10 \times 100 \mathrm{~ms})$ the saturation pulse lasts. Note that the $c_{2}$ profiles (Fig. 6, especially the M55 traces) display a sharp decrease during the first $100 \mathrm{~ms}$ of each pulse, supporting the idea of rapid closure of PSII RCs followed by an $F_{\mathrm{M}}$ level that remains fairly constant during the last $900 \mathrm{~ms}$ - the level used to give an estimate of the $F_{\mathrm{V}}$ values in Table S3. This average $F_{\mathrm{M}}$ level during the pulse is in turn attributed to the relative concentration of the species. Thus, given $F_{\mathrm{V}}$ values that progressively decrease, we infer indeed that the concentration of the species effectively decreases over time. Take the case for the M55 mutant after 34 min DA (red in Fig. 6) as an example: notice how the effect of the saturation pulses on the sample evolves from (times $t<50 \mathrm{~s}$ ) raising the $F_{\mathrm{M}}$ level above the $F_{\mathrm{S}}$ level (positive $F_{\mathrm{V}}$ value) to (times $t>50 \mathrm{~s}$ ) not having virtually any other effect than the rapid closure of RCs within the first $100 \mathrm{~ms}$ of the pulse, after which the fluorescence level during saturation matches that of the $F_{\mathrm{S}}$ levels during background illumination $\left(F_{\mathrm{V}}\right.$ value is virtually zero). Furthermore, the $F_{\mathrm{V}}$ decrease we have just described is accompanied by an $F_{\mathrm{V}}$ increase in the $c_{3}$ profile, as visible in Fig. 7 (concentration profiles of PB-PSII-PSI complexes with slow PB $\rightarrow$ PSI energy transfer): note how the $\mathrm{DA}=34 \mathrm{~min}$ profile (red) systematically has lower $F_{\mathrm{M}}$ levels for times $t<50 \mathrm{~s}$ but gradually increases until it, for times $t>50 \mathrm{~s}$, has reached the same $F_{\mathrm{M}}$ levels as the rest of the traces-the DA $=21$ min profile (orange) being an interesting exception.

Therefore, although the $F_{\mathrm{S}}$ levels always correspond to a mixture of hybrid species, the $F_{\mathrm{M}}$ levels allow one to make a statement on the relative concentration of the species with closed RCs (Fig. 1e, g). Considering that the $F_{\mathrm{S}}$ levels of $c_{1}$ remain relatively constant throughout the experiment with average $F_{\mathrm{V}}$ values of -0.35 for both M55 and WT (cf. Table S3), we conclude that a population exchange exclusively exists between the species with fast and slow EET to PSI.

Finally, this effect on the $F_{\mathrm{V}}$ values also clearly depends on the length of the DA periods. Compare, for instance, in the WT traces in Fig. 6, the $F_{\mathrm{V}}$ values of the second pulse for $\mathrm{DA}=1$ and $\mathrm{DA}=34 \mathrm{~min}$ : 0.04 for the former, 0.14 for the latter (cf. $F_{\mathrm{Vi}}$ values in Table S3). This means that a difference of ca. $30 \mathrm{~min}$ in DA has led to $10 \%$ more of the megacomplex with fast PB $\rightarrow$ PSI EET being formed. The formation of these complexes, we propose, is at the core of state transitions. Figure 7, showing the $c_{3}$ concentration profiles may help visualizing this idea: the first peak illustrates the decreasing concentration of megacomplexes with slow EET to PSI, which we attribute to the state 1 to state 2 transition in darkness, related to respiratory activity. Based on the stoichiometry published by Moal and Lagoutte (2012), who suggest a dense packing of pigment-protein complexes in the thylakoid membranes of Synechocystis, we speculate that a conformational change occurs during darkness, which would yield an even denser packing, favoring fast energy PB $\rightarrow$ PSI transfer. Physical re-distribution of the complexes could result in slowing down the EET rate to PSI.

Finally, we come to compare the M55 mutant and the WT on the basis of a summary figure, that we will briefly describe first: Fig. 8 shows, for a selected DA time (34 min), the scaled SASs (Fig. 8a) of WT and M55 as well as the corresponding time traces (Fig. 8b), accompanied by the species that we conclude that the signal originates from. For comparison, $\mathrm{SAS}_{3}$ from $\triangle \mathrm{PSI}$ is also shown, where the small F660/F680 ratio is due to the EET from PB to PSII exclusively, with the closed PSII RC responsible for the intense $\mathrm{Chl} a$ emission that peaks at $680 \mathrm{~nm}$. The spectra in Fig. 8a have been normalized relative to the $640 \mathrm{~nm}$ phycocyanin emission, which is expected to be the same in all samples. The resulting $\triangle$ PSI-SAS confirms the analyses of 

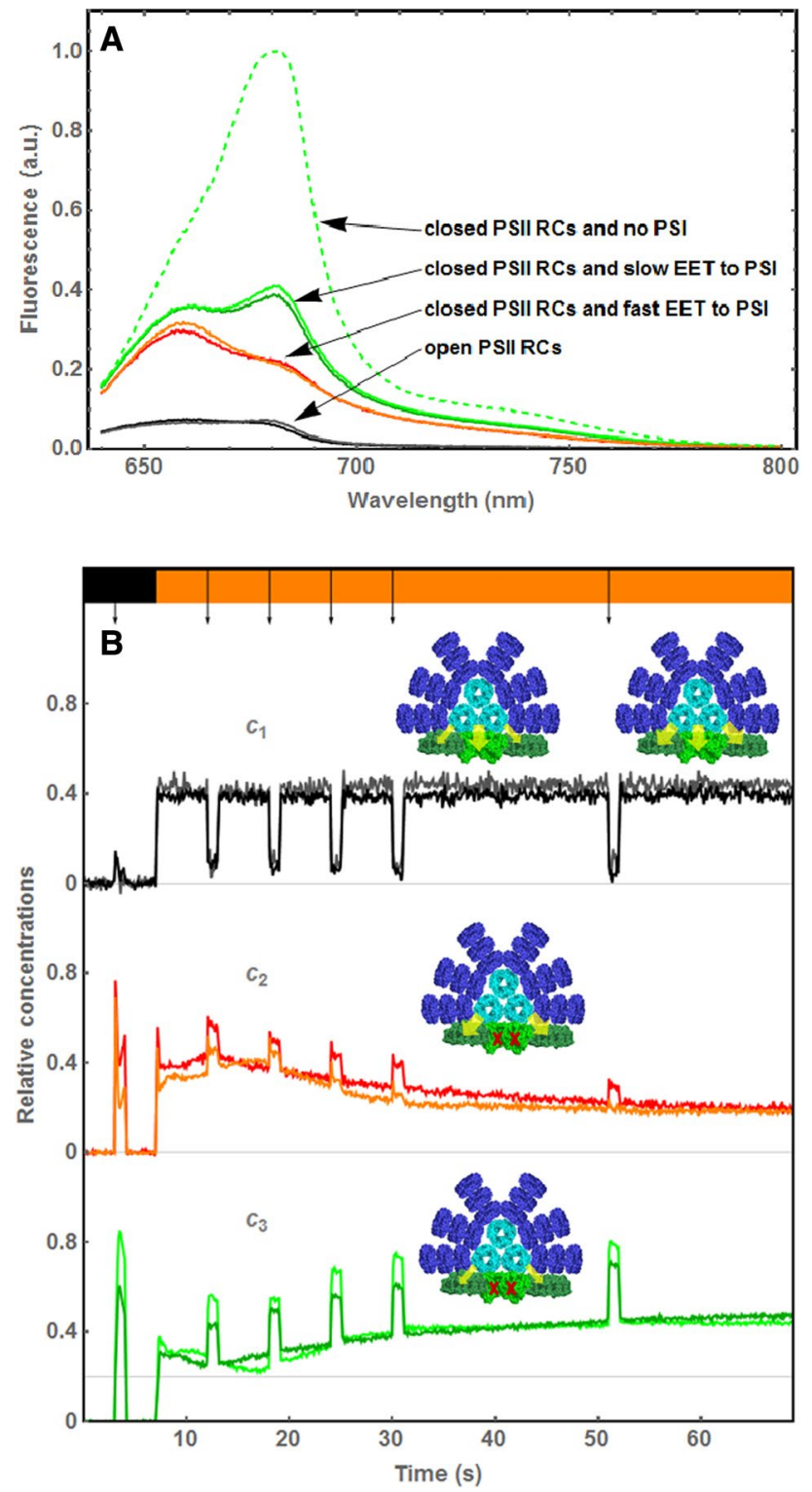

Fig. 8 a Selection of SASs from experiments with $\mathrm{DA}=34 \mathrm{~min}$. The spectra of the WT and M55 have been normalized relative to the PB emission at $640 \mathrm{~nm}$ of the $\triangle$ PSI spectrum. Key green: $\mathrm{SAS}_{3}$ in WT, light green $\mathrm{SAS}_{3}$ in M55, red $\mathrm{SAS}_{2}$ in WT, orange $\mathrm{SAS}_{2}$ in M55, black $\mathrm{SAS}_{1}$ in WT, gray $\mathrm{SAS}_{1}$ in M55, light green-dashed $\mathrm{SAS}_{3}$ in $\triangle$ PSI. b Zoom-in of the first minute of the time profiles. The inferred fluorescent species are shown as an inset

independent measurements in the $\triangle$ PSI mutant carried out in a set-up in Třeboň, CZ (see Fig. 7 in Acuña et al. 2016). As for the time profiles in Fig. 8b, we would like to point at a key difference between the M55 mutant and the WT based upon the two following observations: (i) in $c_{2}$, the $F_{\mathrm{V}}$ values for times $t>50 \mathrm{~s}$ are close to zero for the M55 (orange), whereas the WT (red) still has a positive value and (ii) in $c_{3}$, the $F_{\mathrm{M}}$ values of the first saturation pulse reveal that the WT (dark green) keeps a considerably lower amount of megacomplexes with slow PB $\rightarrow$ PSI energy transfer than the M55 (light green) mutant does. In other words, in darkness, the M55 mutant forms less megacomplexes with fast $\mathrm{PB} \rightarrow$ PSI energy transfer than the WT, while it also, during background illumination, exchanges its population for slow $\mathrm{PB} \rightarrow$ PSI transferring megacomplexes more quickly than the WT does. It is almost as if the M55 mutant resisted to having to form the megacomplex with fast PB $\rightarrow$ PSI EET even though it is capable of doing so. This would be consistent in that, given a dysfunctional NDH-1 and consequently an impaired CEF around PSI and lower input of electrons into the PQ pool, the cell would favor a lower PSI activity by keeping a higher population of PB-PSII-PSI complexes with low EET to PSI (Fig. 1g) instead of the species with high PB-to-PSI EET rate (Fig. 1e). From the use of the M55 mutant, we conclude that the decreased rate of reduction of the PQ-pool-due to the dysfunctional NDH-1 complex-has a clear effect on the state of PSII ( $p q$-vs. $f v$-closure) and shows the entanglement between respiration and photosynthesis in the cyanobacterial thylakoid membrane.

Ultimately, each SAS that we have resolved in this study should be explainable as the steady-state emission spectrum of a complex from Fig. 1. Recently, we have been able to reconstruct the SASs of the $\triangle$ PSI mutant from the spectral properties of the chromophores, the energy transfer kinetics and the photochemical quenching in the PBS-PSII complex (Acuña et al. 2018b). This is a first step towards linking the here observed SASs to molecular events and structures.

\section{Concluding remarks}

We have presented a series of experiments during which Synechocystis WT and mutants thereof undergo a dark-tolight transition after being prepared under different conditions. The data matrices have been analyzed as rank 3 systems. Two major findings are presented: first, from the DCMU experiments we conclude the existence of a pool of PSII electron acceptors additional to the PQ pool and postulate that the Flv2 and Flv4 proteins could build such a pool. Thus, a PSII RC would be fully open (closed) when it is both $p q$ - and $f v$-open (closed). In the DCMU experiments, the $F_{\mathrm{S}}$ levels suggest PSII RCs that are in the state ' $p q$-closed/flv-open' (Fig. 3). Moreover, the PSII $\rightarrow$ Flv2/4 electron transfer mechanism (i) may require a high energy back transfer rate to the $\mathrm{PB}$; and (ii) increases its efficiency with a well-functioning NDH-1 complex; which points to a possible (so far unknown) electron acceptor to the Flv2/4 heterodimer. A fundamental assumption in this reasoning implies that electrons transferred to the Flv2/4 pool bypass the secondary quinone $Q_{\mathrm{B}}$. Second, we postulate that $\mathrm{SAS}_{2}$ in Fig. 5 originates from a PB-PSII-PSI megacomplex with fast EET to PSI and $p q$-closed. A slow EET to PSI results in 
$\mathrm{SAS}_{3}$ in Fig. 5. During darkness, the thylakoid membrane may re-arrange to a more tightly packed ensemble of pigment-protein complexes favoring fast EET of PB to PSI. This would be the state 1 to state 2 transition. With the onset of photosynthetic activity, the pigment-protein complexes re-distribute over the membrane leading to the loosening of the PB-PSI coupling and ultimately slowing down EET. This, in turn, would be the state 2 to state 1 transition. This interpretation aligns well with the generally accepted idea that state transitions are a mechanism by means of which the energy inputs to PSI and PSII are optimized resulting in a low (state 2) and in a high (state 1) fluorescent state. Presumably due to its limited capacity to perform CEF around PSI, the M55 mutant forms less fast PB-to-PSI EET complexes than the WT. With the current spectral and time resolution of the set-up, we do not see any evidence for CpcG2-type of $\mathrm{PB}$, nor uncoupled PB. Furthermore, it is also not possible to resolve PB-PSII-PSI megacomplexes and PB-PSII complexes when PSII RCs are (either $p q$ - or $f v$-)open. Though this study provides important hints of the modus operandi of state transitions, further studies involving multiple excitation wavelengths could help establishing more precisely their molecular triggers.

Acknowledgements AMA gratefully acknowledges Michael Reus for his assistance in re-building the multiple LED set-up in Amsterdam and Joris Snellenburg for his valuable assistance during the automation of the data acquisition as well as helpful discussions. Roberta Croce and Alfred Holzwarth are thanked for letting us use this set-up. The $\Delta$ PSI mutant which was originally constructed by Wim Vermaas (Arizona State University, Tempe, Arizona, U.S.) was kindly provided to us by Christiane Funk (Umeå University, Sweden). M55 was kindly provided by Teruo Ogawa (Nagoya University, Japan). This research was performed as part of the BioSolar Cells research program, sponsored by the Dutch Ministry of Economic Affairs. This work is part of the research program of the Netherlands Organization for Scientific Research (NWO, previously FOM).

Open Access This article is distributed under the terms of the Creative Commons Attribution 4.0 International License (http://creativeco mmons.org/licenses/by/4.0/), which permits unrestricted use, distribution, and reproduction in any medium, provided you give appropriate credit to the original author(s) and the source, provide a link to the Creative Commons license, and indicate if changes were made.

\section{References}

Acuña AM, Kaňa R, Gwizdala M, Snellenburg JJ, van Alphen P, van Oort B, Kirilovsky D, van Grondelle R, van Stokkum IHM (2016) A method to decompose spectral changes in Synechocystis PCC 6803 during light-induced state transitions. Photosynth Res 130(1):237-249

Acuña AM, van Alphen P, Branco dos Santos F, van Grondelle R, Hellingwerf KJ, van Stokkum IHM (2018a) Spectrally decomposed dark-to-light transitions in a PSI-deficient mutant of Synechocystis sp. PCC 6803. Biochim Biophys Acta 1859(2):57-68
Acuña AM, Van Alphen P, Van Grondelle R, Van Stokkum IHM (2018b) The phycobilisome terminal emitter transfers its energy with a rate of (20 ps)-1 to photosystem II. Photosynthetica 56(1):265-274

Allahverdiyeva Y, Mustila H, Ermakova M, Bersanini L, Richaud P, Ajlani G, Battchikova N, Cournac L, Aro E-M (2013) Flavodiiron proteins Flv1 and Flv3 enable cyanobacterial growth and photosynthesis under fluctuating light. Proc Natl Acad Sci USA 110(10):4111-4116

Allahverdiyeva Y, Isojärvi J, Zhang P, Aro E-M (2015) Cyanobacterial oxygenic photosynthesis is protected by flavodiiron proteins. Life 5(1):716

Bersanini L, Battchikova N, Jokel M, Rehman A, Vass I, Allahverdiyeva Y, Aro E-M (2014) Flavodiiron protein Flv2/Flv4-related photoprotective mechanism dissipates excitation pressure of PSII in cooperation with phycobilisomes in cyanobacteria. Plant Physiol 164(2):805-818

Bersanini L, Allahverdiyeva Y, Battchikova N, Heinz S, Lespinasse M, Ruohisto E, Mustila H, Nickelsen J, Vass I, Aro E-M (2017) Dissecting the photoprotective mechanism encoded by the flv4-2 operon: a distinct contribution of S110218 in photosystem II stabilization. Plant Cell Environ 40(3):378-389

Björn LO, Nickelsen K (2013) Evolution of the Z-scheme of electron transport in oxygenic photosynthesis. In: Photosynthesis research for food, fuel and the future. Springer, Berlin, pp 827-833

Campbell D, Hurry V, Clarke AK, Gustafsson P, Öquist G (1998) Chlorophyll fluorescence analysis of cyanobacterial photosynthesis and acclimation. Microbiol Mol Biol Rev 62(3):667-683

Chen Q (2017) Engineering retinal-based phototrophy via a complementary photosystem in Synechocystis sp. PCC6803. PhD thesis, University of Amsterdam, Amsterdam

Chidgey JW, Linhartova M, Komenda J, Jackson PJ, Dickman MJ, Canniffe DP, Konik P, Pilny J, Hunter CN, Sobotka R (2014) A cyanobacterial chlorophyll synthase-HliD complex associates with the Ycf39 protein and the YidC/Alb3 insertase. Plant cell 26(3):1267-1279

Chukhutsina V, Bersanini L, Aro E-M, van Amerongen H (2015) Cyanobacterial light-harvesting phycobilisomes uncouple from photosystem I during dark-to-light transitions. Sci Rep 5:14193

Cooley JW, Vermaas WFJ (2001) Succinate dehydrogenase and other respiratory pathways in thylakoid membranes of Synechocystis sp. strain PCC 6803: capacity comparisons and physiological function. J Bacteriol 183(14):4251-4258

Dong C, Tang A, Zhao J, Mullineaux CW, Shen G, Bryant DA (2009) $\mathrm{ApcD}$ is necessary for efficient energy transfer from phycobilisomes to photosystem I and helps to prevent photoinhibition in the cyanobacterium Synechococcus sp. PCC 7002. Biochim Biophys Acta 1787(9):1122-1128

Ermakova M, Huokko T, Richaud P, Bersanini L, Howe CJ, Lea-Smith DJ, Peltier G, Allahverdiyeva Y (2016) Distinguishing the roles of thylakoid respiratory terminal oxidases in the cyanobacterium Synechocystis sp. PCC 6803. Plant Physiol 171(2):1307-1319

Gao F, Zhao J, Chen L, Battchikova N, Ran Z, Aro E-M, Ogawa T, Ma W (2016) The NDH-1L-PSI supercomplex is important for efficient cyclic electron transport in cyanobacteria. Plant Physiol 172(3):1451-1464

Golub G, Van Loan C (1996) The singular value decomposition and unitary matrices. Matrix Comput 1996:70-71

Govindjee, Shevela D (2011) Adventures with cyanobacteria: a personal perspective. Front Plant Sci 2:28

Jallet D, Gwizdala M, Kirilovsky D (2012) ApcD, ApcF and ApcE are not required for the orange carotenoid protein related phycobilisome fluorescence quenching in the cyanobacterium Synechocystis PCC 6803. Biochim Biophys Acta 1817(8):1418-1427 
Jeanjean R, Matthijs HC, Onana B, Havaux M, Joset F (1993) Exposure of the cyanobacterium Synechocystis PCC6803 to salt stress induces concerted changes in respiration and photosynthesis. Plant Cell Physiol 34(7):1073-1079

Kaňa R, Prášil O, Komárek O, Papageorgiou GC, Govindjee (2009) Spectral characteristic of fluorescence induction in a model cyanobacterium, Synechococcus sp. (PCC 7942). Biochim Biophys Acta 1787(10):1170-1178

Kaňa R, Kotabová E, Komárek O, Šedivá B, Papageorgiou GC, Govindjee, Prášil O (2012) The slow S to M fluorescence rise in cyanobacteria is due to a state 2 to state 1 transition. Biochim Biophys Acta 1817(8):1237-1247

Kirilovsky D (2007) Photoprotection in cyanobacteria: the orange carotenoid protein (OCP)-related non-photochemical-quenching mechanism. Photosynth Res 93(1-3):7

Kirilovsky DL, Vernotte C, Etienne AL (1990) Protection from photoinhibition by low temperature in Synechocystis 6714 and in Chlamydomonas reinhardtii: detection of an intermediary state. Biochemistry 29(35):8100-8106

Kirilovsky D, Kaňa R, Prášil O (2014) Mechanisms modulating energy arriving at reaction centers in cyanobacteria. In: Demmig-Adams B, Garab G, Adams W III, Govindjee (eds) Non-photochemical quenching and energy dissipation in plants, algae and cyanobacteria. Advances in photosynthesis and respiration, vol 40. Springer, Dordrecht, pp 471-502

Kondo K, Mullineaux CW, Ikeuchi M (2009) Distinct roles of CpcG1phycobilisome and $\mathrm{CpcG} 2$-phycobilisome in state transitions in a cyanobacterium Synechocystis sp. PCC 6803. Photosynth Res 99(3):217-225

Lambrev PH, Nilkens M, Miloslavina Y, Jahns P, Holzwarth AR (2010) Kinetic and spectral resolution of multiple nonphotochemical quenching components in arabidopsis leaves. Plant Physiol 152(3):1611-1624

Lea-Smith DJ, Bombelli P, Vasudevan R, Howe CJ (2016) Photosynthetic, respiratory and extracellular electron transport pathways in cyanobacteria. Biochim Biophys Acta 1857(3):247-255

Liu L-N (2015) Distribution and dynamics of electron transport complexes in cyanobacterial thylakoid membranes. Biochim Biophys Acta 1857(3):256-265

Liu L-N, Bryan SJ, Huang F, Yu J, Nixon PJ, Rich PR, Mullineaux CW (2012) Control of electron transport routes through redoxregulated redistribution of respiratory complexes. Proc Natl Acad Sci USA 109(28):11431-11436

Liu H, Zhang H, Niedzwiedzki DM, Prado M, He G, Gross ML, Blankenship RE (2013) Phycobilisomes supply excitations to both photosystems in a megacomplex in cyanobacteria. Science 342(6162):1104-1107

Mi H, Endo T, Ogawa T, Asada K (1995) Thylakoid membrane-bound, NADPH-specific pyridine nucleotide dehydrogenase complex mediates cyclic electron transport in the cyanobacterium Synechocystis sp. PCC 6803. Plant Cell Physiol 36(4):661-668

Moal G, Lagoutte B (2012) Photo-induced electron transfer from photosystem I to NADP+: characterization and tentative simulation of the in vivo environment. Biochim Biophys Acta 1817(9):1635-1645

Mullineaux CW, Allen JF (1986) The state 2 transition in the cyanobacterium Synechococcus 6301 can be driven by respiratory electron flow into the plastoquinone pool. FEBS Lett 205(1):155-160

Mullineaux CW, Allen JF (1990) State 1-state 2 transitions in the cyanobacterium Synechococcus 6301 are controlled by the redox state of electron carriers between photosystems I and II. Photosynth Res 23(3):297-311
Mullineaux CW, Emlyn-Jones D (2005) State transitions: an example of acclimation to low-light stress. J Exp Bot 56(411):389-393

Mullineaux CW, Holzwarth AR (1993) Effect of photosystem II reaction centre closure on fluorescence decay kinetics in a cyanobacterium. Biochim Biophys Acta 1183(2):345-351

Ogawa T (1991) A gene homologous to the subunit-2 gene of NADH dehydrogenase is essential to inorganic carbon transport of Synechocystis PCC6803. Proc Natl Acad Sci USA 88(10):4275-4279

Ohkawa H, Price GD, Badger MR, Ogawa T (2000) Mutation of ndh genes leads to inhibition of $\mathrm{CO}_{2}$ uptake rather than $\mathrm{HCO}_{3}-$ uptake in Synechocystis sp. strain PCC 6803. J Bacteriol 182(9):2591-2596

Papageorgiou GC (1996) The photosynthesis of cyanobacteria (blue bacteria) from the perspective of signal analysis of chlorophyll $a$ fluorescence. J Sci Ind Res 55(8-9):596-617

Schmetterer G (1994) Cyanobacterial respiration. In: The molecular biology of cyanobacteria. Springer, Dordrecht, pp 409-435

Schuurmans RM, van Alphen P, Schuurmans JM, Matthijs HCP, Hellingwerf KJ (2015) Comparison of the photosynthetic yield of cyanobacteria and green algae: different methods give different answers. PLoS ONE 10(9):e0139061

Shen G, Boussiba S, Vermaas WFJ (1993) Synechocystis sp. PCC 6803 strains lacking photosystem I and phycobilisome function. Plant Cell 5(12):1853-1863

Shimakawa G, Shaku K, Nishi A, Hayashi R, Yamamoto H, Sakamoto K, Makino A, Miyake C (2015) Flavodiiron2 and flavodiiron4 proteins mediate an oxygen-dependent alternative electron flow in Synechocystis sp. PCC 6803 under $\mathrm{CO}_{2}$-limited conditions. Plant Physiol 167(2):472-480

Sinha RK, Komenda J, Knoppová J, Sedlářová M, Pospíšil P (2012) Small CAB-like proteins prevent formation of singlet oxygen in the damaged photosystem II complex of the cyanobacterium Synechocystis sp. PCC 6803. Plant Cell Environ 35(4):806-818

Stirbet A, Govindjee (2011) On the relation between the Kautsky effect (chlorophyll a fluorescence induction) and photosystem II: basics and applications of the OJIP fluorescence transient. J Photochem Photobiol B 104:236-257

Trautmann D, Voß B, Wilde A, Al-Babili S, Hess WR (2012) Microevolution in cyanobacteria: re-sequencing a motile substrain of Synechocystis sp. PCC 6803. DNA Res 19(6):435-448

van Grondelle R, Dekker JP, Gillbro T, Sundstrom V (1994) Energy transfer and trapping in photosynthesis. Biochim Biophys Acta 1187(1):1-65

van Thor JJ, Gruters OW, Matthijs HC, Hellingwerf KJ (1999) Localization and function of ferredoxin: NADP + reductase bound to the phycobilisomes of Synechocystis. EMBO J 18(15):4128-4136

Vermaas WFJ, Shen G, Styling S (1994) Electrons generated by photosystem II are utilized by an oxidase in the absence of photosystem I in the cyanobacterium Synechocystis sp. PCC 6803. FEBS Lett 337(1):103-108

Zavřel T, Očenášová P, Červený J (2017) Phenotypic characterization of Synechocystis sp. PCC 6803 substrains reveals differences in sensitivity to abiotic stress. PLoS ONE 12(12):e0189130

Zhang P, Allahverdiyeva Y, Eisenhut M, Aro E-M (2009) Flavodiiron proteins in oxygenic photosynthetic organisms: photoprotection of photosystem II by Flv2 and Flv4 in Synechocystis sp. PCC 6803. PLoS ONE 4(4):e5331

Zlenko DV, Galochkina TV, Krasilnikov PM, Stadnichuk IN (2017) Coupled rows of PBS cores and PSII dimers in cyanobacteria: symmetry and structure. Photosynth Res 133(1-3):245-260 